\title{
Theorizing Probability Distribution in Applied Statistics
}

Indra Malakar (M. Phil.)*

\begin{abstract}
This paper investigates into theoretical knowledge on probability distribution and the application of binomial, poisson and normal distribution. Binomial distribution is widely used discrete random variable when the trails are repeated under identical condition for fixed number of times and when there are only two possible outcomes whereas poisson distribution is for discrete random variable for which the probability of occurrence of an event is small and the total number of possible cases is very large and normal distribution is limiting form of binomial distribution and used when the number of cases is infinitely large and probabilities of success and failure is almost equal.
\end{abstract}

Key words: Application, binomial, discrete, normal, occurrence \& poisson.

\section{Introduction}

In statistics, probability theory and probability distribution are mathematical functions that provide the probabilities of occurrence of different possible outcomes in an experiment (Ash, 2008). In more technical terms, the probability distribution is a description of a random phenomenon in terms of the probabilities of events (Michael, 2010). A probability distribution is specified in terms of an underlying sample space, which is the set of all possible outcomes of the random phenomenon being observed. The sample space may be the set of real numbers or a set of vectors, or it may be a list of non-numerical values; for example, the sample space of a coin flip would be (heads \& tails). Probability distributions are generally categorized into two classes. A discrete probability distribution (applicable to the scenarios where the set of possible outcomes is discrete, such as a coin toss or a roll of dice) can be encoded by a discrete list of the probabilities of the outcomes, known as a probability mass function. A continuous probability distribution (applicable to the scenarios where the set of possible outcomes can take on values in a continuous

*Mr. Malakar is a Lecturer at the Department of Population Studies, Patan Multiple Campus, TU, Lalitpur, Nepal. Email:indraamali1@yahoo.com 
range i.e. real numbers, such as the temperature on a given day) is typically described by probability density functions.

The normal distribution is a commonly encountered continuous probability distribution. A probability distribution whose sample space is one-dimensional (for example real numbers, list of labels, ordered labels or binary) is called univariate, while a distribution whose sample space is a vector space of dimension two or more is called multivariate. A univariate distribution gives the probabilities of a single random variable taking on various alternative values; a multivariate distribution (a joint probability distribution) gives the probabilities of a random vector-a list of two or more random variables-taking on various combinations of values. Important and commonly encountered univariate probability distributions include the binomial distribution, the hyper geometric distribution, and the normal distribution. The multivariate normal distribution is a commonly encountered multivariate distribution (Khuri, 2004).

In course of teaching applied mathematics in master's degree semester level in social sciences, most of the learners are found unfamiliar with the usages of statistical tools for data analysis. And even if the questions are viewed in semester level of different social sciences especially in applied statistics then questions targeted to make the learners practice and learn statistical tools so that they can easily interpret and analyze the collected data to give the concrete and meaningful result. So this study can be helpful to use probability distribution in applied statistics with high degree of consistency.

\section{Objectives}

The main objective of this study is to analyze the theoretical knowledge of probability distribution. The specific objectives are to provide the basic concepts of probability distribution and to assess the application of probability distribution in applied statistics to the readers.

\section{Methodology}

The information in this research study has been taken from the secondary sources. The collective data and descriptive information are presented in different tables and some examples of application of probability distribution.

\section{Analysis and Interpretation of the Data}

To define probability distributions for the simplest cases, it is necessary to define random variable and distinguishes between discrete and continuous random variables. Definition 
of probability distribution, mathematical expectation, types of theoretical distribution among which binomial distribution, poisson distribution and normal distribution and their applications have been described and some examples have been solved so that learners can understand the terms discussed.

\section{Random Variable}

A set of numerical values assigned to all possible outcomes of the random experiments are called random variables. If we write $\mathrm{A}, \mathrm{B} . . ., \mathrm{F}$ on six faces of a dice, these letters are not random variables (r.v). If we write some numerical values on the six faces of a die like $1,2,3 \ldots, 6$. We have a set of values called random variable. For example we are going to study on status of Newari and Chhetri people in a certain place and we are selecting two people from a group of Newari and Chhetri family. Let $\mathrm{N}$ stands for Newari people and $\mathrm{C}$ stands for Chhetri people. If we select two people then there are two possible outcomes which are NN, NC, CN and CC. Each outcome can be assigned some numerical values. Let us count number of Newari people in each outcome.

\begin{tabular}{|l|c|}
\hline Outcome & No. of Newari people \\
\hline $\mathrm{CC}$ & 0 \\
\hline $\mathrm{CN}$ & 1 \\
\hline $\mathrm{NC}$ & 1 \\
\hline $\mathrm{NN}$ & 2 \\
\hline
\end{tabular}

Thus the numerical values $0,1,2$ are the values of the random variable where random variable is the number of Newari people in this discussion. A random variable is denoted by a capital letter $\mathrm{X}$. Here $\mathrm{X}$ is the number of Newari people. A random variable is also called chance variable. If we have two or more than two random variables we can use the letters $\mathrm{X}, \mathrm{Y}, \mathrm{Z}$ for them. A random variable may be discrete or continuous.

\section{Discrete Random Variable}

A random variable $X$ is called discrete if it can assume finite number of value. If two people are selected from the Newari and Chhetri family from certain place the number of Newari people selection may be 0,1 or 2 . The range of the variable is from 0 to 2 and random variable can take some selected values in the range. The number of Newari people cannot be 0.1 or 1.5 . This random variable can take only the specific values 
which are 0,1 and 2. For example: a number of children per women, a number of users that use any family planning method.

\section{Continuous Random Variable}

A random variable $X$ is called continuous if it can assume all possible values in the possible range of the random variable. Suppose the temperature in a certain city in the month of June in the past year has always been between "35 degree to 45 degrees" centigrade. The temperature on any day may be $40.15{ }^{\circ} \mathrm{C}$ or $40.16{ }^{\circ} \mathrm{C}$ or it may take any value between $40.15{ }^{\circ} \mathrm{C}$ and $40.16{ }^{\circ} \mathrm{C}$. When we say that the temperature lies between somewhere between $49.5{ }^{\circ} \mathrm{C}$ to $40.5{ }^{\circ} \mathrm{C}$, it is continuous random variable. In discrete variable the values of the variable are exact like 0,1 , and 2 . In continuous random variable the value of the variable is never exact point. It is always in the form of an interval, the interval may be very small. For example a woman's reproductive period is a continuous random variable because it may take any value in the interval 15 to 49 .

\section{Probability Distribution of Random Variable}

Probability distribution of a random variable is a listing of the various values of random variable with their corresponding probabilities. The probability distribution describes how the outcomes of a random experiment are expected to vary. It is also known as the theoretical frequency or relative frequency distribution. It constitutes the breakdown of the total probability. Since a random variable has to assume one of its values, the sum of probabilities in a probability distribution must be 1 . If $x_{1}, x_{2}, \ldots . ., x_{n}$ are the values of the random variable "X" with respective probabilities $P_{1}, P_{2}, \ldots . ., P_{n}$ such that $P_{1}+P_{2}+\ldots P_{n}$ $=1$ and $0 \leq \mathrm{p} \leq 1$. Then the probability distribution of the random variable " $\mathrm{X}$ " is defined as $\mathrm{P}(\mathrm{X}=\mathrm{x})=\mathrm{p}(\mathrm{x})$ and $0 \leq \mathrm{p} \leq 1$.

\begin{tabular}{|l|l|l|l|l|}
\hline Random variable $(\mathrm{X})$ & $x_{1}$ & $x_{2}$ & $x_{3}$ & $x_{4}$ \\
\hline Probability & $P_{1}$ & $P_{2}$ & $P_{3}$ & $P_{4}$ \\
\hline
\end{tabular}

\section{Mathematical Expectation}

The mathematical expectation of a random variable is the average of probability distribution. Or the sum of the product of the various values of random variable and their respective probabilities is mathematical expectation of the random variable. Then the mathematical expectation of the random variable " $\mathrm{X}$ " denoted by $\mathrm{E}(\mathrm{X})$ and is defined as: 
For discrete random variable, $\mathrm{E}(\mathrm{X})=P_{1} x_{1}+P_{2} x_{2}+\ldots \ldots \ldots \ldots P_{n} x_{n}=\sum X . P(x), \mathrm{E}\left(x^{2}\right)$ $=P_{1} x_{1}^{2}+P_{2} x_{2}^{2}+\ldots \ldots \ldots \ldots \ldots . . . P_{n} x_{n}^{2}=\sum x^{2} . \mathrm{P}(\mathrm{x})$

For continuous random variable, $\mathrm{E}(\mathrm{X})=\int_{-\infty}^{\infty} X \mathrm{~F}(\mathrm{X}) \mathrm{dx}, \mathrm{E}(\mathrm{X})=\int_{-\infty}^{\infty} x^{2} \mathrm{~F}(\mathrm{X}) \mathrm{dx}$ where $\mathrm{E}(\mathrm{X})=\int_{-\infty}^{\infty} X \mathrm{~F}(\mathrm{X}) \mathrm{dx}=1 ; \mathrm{F}(\mathrm{x}) \geq 0$.

Therefore, Mean $(\mathrm{X})=\mathrm{E}(\mathrm{X})$ and Variance $\left(\sigma^{2}\right)=\mathrm{E}\left(X^{2}\right)-[E(X)]^{2}$.

Example of mathematical expectation: Suppose two urns contain respectively 5 white and 3 black balls and 2 white and 3 black balls respectively. One ball is drawn from each urn and the expected number of white ball drawn can be determined as : If $\mathrm{W}$ and $\mathrm{B}$ denote white and black ball, $\mathrm{W}_{1}, \mathrm{~B}_{1}$ and $\mathrm{W}_{2}, \mathrm{~B}_{2}$ be the balls in first and second urn respectively then

\begin{tabular}{|c|c|}
\hline $\begin{array}{cc}\text { First Urn } & 5 w_{1} \\
& 3 B_{1} \\
\end{array}$ & $\begin{array}{r}\text { Second Urn } 2 w_{2} \\
3 B_{2} \\
\end{array}$ \\
\hline Outcomes & Probabilities \\
\hline$w_{1} w_{2}$ & $\frac{5}{8} x \frac{2}{5}=10 / 40$ \\
\hline$w_{1} B_{2}$ & $\frac{5}{8} x \frac{3}{5}=15 / 40$ \\
\hline$w_{2} B_{1}$ & $\frac{2}{5} \times \frac{3}{8}=6 / 40$ \\
\hline$B_{1} B_{2}$ & $\frac{3}{8} x \frac{3}{5}=9 / 40$ \\
\hline
\end{tabular}

Now, No white ball $(\mathrm{X}=0), \mathrm{P}(0)=\mathrm{P}\left(B_{1} B_{2}\right)=9 / 40$. One white ball (one white \& one black $), \mathrm{P}(1)=\mathrm{P}\left(w_{1} B_{2}\right)+\mathrm{P}\left(w_{2} B_{1}\right)=15 / 40+6 / 40=21 / 40$.

Two white balls, $\mathrm{P}(2)=\mathrm{P}\left(w_{1} w_{2}\right)=10 / 40$. Now probability distribution of white balls

\begin{tabular}{|l|l|l|}
\hline$X$ & $P(x)$ & $X P(x)$ \\
\hline 0 & $9 / 40$ & 0 \\
\hline 1 & $21 / 40$ & $21 / 40$ \\
\hline 2 & $10 / 40$ & $20 / 40$ \\
\hline $\mathrm{E}(\mathrm{x})$ & & $0+21 / 40+20 / 40=41 / 40$ \\
\hline
\end{tabular}

So, the expected number of white balls $=41 / 40$. 


\section{Types of Theoretical Distribution}

- Discrete theoretical distribution

a. Binomial distribution

b. Poisson distribution

- Continuous distribution

a. Normal distribution

\section{Binomial Distribution}

It is widely used probability distribution of discrete random variable introduced by James Bernoulli in 1705 A.D. It describes the possible number of time that a particular event will occur in a sequence of distribution which has four conditions.

Conditions of Binomial Distribution:

- Trials are repeated under identical condition for fixed number of times i.e. the number of trials are finite and fixed e.g. if a die is rolled 6 times, then the number of trials $(\mathrm{n})=6$.

- There are only two possible outcomes, success or failure, lose or win, head or tail for each trial e.g. if student appears in exam then he will either pass or fail the exam.

- The probability of success in each trial remains constant and does not change from trial to trial e.g. if a coin is tossed 6 times then the probability of tail in each trial is $1 / 2$.

- The trials are independent i.e. the probability of an event in any trial is not affected by the results of any other trials e.g. if a coin is tossed, the probability of appearing tail does not affect the occurrence of the tail and vice versa.

\section{Properties of Binomial Distribution}

- Since it assumes only positive integers values like $0,1,2, \ldots$, binomial distribution is a discrete probability distribution.

- Binomial distribution is known as bi-parametric distribution as it is characterized by two parameters $n$ and $p$. If the values of $n$ and $p$ are known then the distribution is known completely.

- The mean of binomial distribution is $\mathrm{X}=\mathrm{E}(\mathrm{X})=\mathrm{np}$. 
- The variance of binomial distribution (standard deviation) is $\operatorname{Var}(\mathrm{X})=\sigma_{x}^{2}=\mathrm{npq}$. Since $\mathrm{p}$ and $\mathrm{q}$ are numerically less than or equal to 1 , npq less than to $\mathrm{np}$ i.e. variance is less than mean.

- Depending on the values of two parameters, binomial distribution may be unmodal or bimodal.

- Skewness $=\frac{(q-p)^{2}}{n p q} ;$ Kurtosis $=3+\frac{1-6 p q}{n p q}$

\section{Application of Binomial Distribution}

It is applicable when the trials are independent and each trial has just two mutually exclusive outcomes success and failures. It is applied in coin toss experiment, sampling inspection plan, genetic experiments, and quality control and so on.

\section{Definition}

A random variable $\mathrm{X}$ is said to have binomial distribution if its probability function is given by $\mathrm{f}(\mathrm{x})=\mathrm{b}(\mathrm{x} ; \mathrm{n}, \mathrm{p})=\mathrm{p}(\mathrm{r})=\mathrm{n} \mathrm{C} \mathrm{r} p^{r} q^{n-r}=\frac{n !}{r !(n-r) !} p^{r} q^{n-r}$ where $\mathrm{x}=0,1,2, \ldots \mathrm{n} . \mathrm{q}$ $=1-\mathrm{p}$ and $\mathrm{n}$ and $\mathrm{p}$ are the parameters and $\mathrm{x}$ is the variable (Feller, 1968). Example: A fair coin is tossed five times then finding the probability of getting i. exactly one head ii. At least one head can be determined as: Let $\mathrm{X}$ be the number of heads in the five tosses, $A$ and $B$ be the probability of the event in which exactly one head and at least one head occurs.

i) $\mathrm{P}(\mathrm{A})=\mathrm{P}(\mathrm{X}=1)=\mathrm{C}(\mathrm{n}, \mathrm{k}) \cdot p^{k} q^{n-k}=\mathrm{c}(5,1)\left(\frac{1}{2}\right)\left(\frac{1}{2}\right)^{5-1}=5 / 32$

ii) At least one head $=$ (one head should be there or 2 heads is also ok or three heads is also ok or 4 heads is also ok or 5 heads is also ok).

$\mathrm{P}(\mathrm{B})=\mathrm{P}($ at least one head $)=(5,1) p^{1} q^{5-1}+\mathrm{c}(5,2) p^{2} q^{5-2}+\mathrm{c}(5,3) p^{3} q^{5-3}+$ $\mathrm{c}(5,4) p^{4} q^{5-4}+\mathrm{c}(5,5) p^{5} q^{5-5}=31 / 32$

OR for $\mathrm{P}(\mathrm{B})$,At least one head means it is the complement of the event $(\mathrm{X}=0)$, so, $\mathrm{P}(\mathrm{B})=1-\mathrm{P}(\mathrm{X}=0)=1-\mathrm{c}(5,0)\left(\frac{1}{2}\right)^{0}\left(\frac{1}{2}\right)^{5}=1-1 / 32=31 / 32$.

\section{Characteristics of Binomial Distribution}

- It has two parameters $\mathrm{n}$ and $\mathrm{p}$. Having $\mathrm{n}$ and $\mathrm{p}$ then probability of all random variable can be introduced.

- In binomial distribution mean is always greater than variance. 
- It is symmetrical if $p=1 / 2$.

- It is positive skewed if $p<1 / 2$. And negative skewed if $p>1 / 2$.

- In binomial distribution mean $=\mathrm{np}$, variance $=\mathrm{npq}$

Expected frequency of binomial distribution

$$
\mathrm{E}=\mathrm{Np}(\mathrm{r})=\mathrm{N} \cdot \mathrm{C}(\mathrm{n}, \mathrm{r}) \cdot p^{r} q^{n-r}
$$

\section{Fitting of Binomial Distribution}

The process of finding the expected or theoretical frequencies on the basis of available information supplied by observed or experimental frequency is called fitting of a distribution. Therefore, the process of finding the expected or theoretical frequencies for a binomial distribution is called fitting of binomial distribution. If random experiment consists of " $n$ " trials satisfying conditions of binomial distribution is repeated $\mathrm{N}$ times than expected frequency of getting exactly ' $r$ ' success is given by $\mathrm{f}(\mathrm{r})=\mathrm{N}$ x $\mathrm{P}(\mathrm{X}=\mathrm{r})=\mathrm{N} x$ $\mathrm{C}(\mathrm{n}, \mathrm{r}) p^{r} q^{n-r}$ (Kolmogorov, 1956). For example: 5 coins are tossed 3200 times and finding the expected frequencies of the distribution of head and tail and also calculating mean number of success and standard deviation can be done as: Here, $n=5$. Total number of trials $(\mathrm{N})=3200$, Probability of getting or success $(\mathrm{P})=0.5$, Probability of getting tail or failure $(\mathrm{q})=1-0.5=0.5$. The expected or theoretical frequencies of getting ' $\mathrm{r}$ ' heads is denoted by $\mathrm{f}(\mathrm{r})$ and is given by $\mathrm{f}(\mathrm{r})=\mathrm{N} X \mathrm{P}(\mathrm{X}=\mathrm{r})=\mathrm{N} \times \mathrm{C}(\mathrm{n}, \mathrm{r}) p^{r} q^{n-r}$ $; \mathrm{r}=0,1,2, \ldots, 5 . \mathrm{f}(\mathrm{r})=3200 \times \mathrm{C}(5, \mathrm{r}) \times(0.5)^{r} \times(0.5)^{5-r}$. Now, when $\mathrm{r}=0, \mathrm{f}(\mathrm{o})=3200 \mathrm{x}$ $\mathrm{c}(5,0) \quad \mathrm{x}(0.5)^{0} \times(0.5)^{5-0}=100$, when $\mathrm{r}=1, \mathrm{f}(1)=3200 \quad \mathrm{x} \quad \mathrm{c}(5,1)$ $\mathrm{x}(0.5)^{1} \times(0.5)^{5-1}=500$, when $\mathrm{r}=2, \mathrm{f}(2)=3200 \mathrm{xc}(5,2) \times(0.5)^{2} \times(0.5)^{5-2}=1000$, when $r=3, f(3)=3200 \times c(5,3) \times(0.5)^{3} \times(0.5)^{5-3}=1000$, when $r=4, f(4)=3200 x$ $\mathrm{c}(5,4) \times(0.5)^{4} \times(0.5)^{5-4}=500$, when $\mathrm{r}=5, \mathrm{f}(5)=3200 \times \mathrm{c}(5,5) \times(0.5)^{5} \times(0.5)^{5-5}=$ 100 . Hence the expected frequency distribution of heads and tails is

\begin{tabular}{|l|l|l|l|l|l|l|}
\hline No. of heads(X) & 0 & 1 & 2 & 3 & 4 & 5 \\
\hline $\begin{array}{l}\text { Expected } \\
\text { frequency }\end{array}$ & 100 & 500 & 1000 & 1000 & 500 & 100 \\
\hline
\end{tabular}

Again, Mean number of success $=\mathrm{n} \mathrm{p}=5 \times 0.5=2.5$

Standard deviation $=\sqrt{n p q}=\sqrt{5 \times 0.5 \times 0.5}=1.118$. 


\section{Poisson Distribution}

Poisson distribution tells about the behavior of discrete random variables for which the probability of occurrence of an event is small and the total number of possible cases is very large. It is a tool that aids to predict the probability of certain events from happening when we know how often the event has occurred. Also, it gives the probability of a given number of events happening in a fixed interval of time.

Poisson distribution like binomial distribution cannot be deduced on purely theoretical grounds based on the conditions of the experiments. In fact, it must be based on experience, i.e. on the empirical results of past experiments relating to the problem under study. The Poisson distribution is the good approximation of the binomial distribution when $\mathrm{n}$ is greater than or equal to 20 and the probability of success for each trial is less than or equal to 0.05 . This distribution deals with the evaluation of probabilities of rare events such as "no. of car accidents", "no. of earthquakes in a year", "no. of printing mistakes per page", "no. of tsunami in a year" and so on.

\section{Condition of Poisson Distribution}

Poisson distribution is limiting form of the binomial distribution under the following conditions,

- When the number of trials ' $n$ ' become sufficiently large, $n \rightarrow \infty$.

- When the probability of success ' $\mathrm{p}$ ' in a trial is very small, $\mathrm{p} \rightarrow 0$

- $\mathrm{np}=\lambda$ (say) is finite positive constant. $\mathrm{np}=\lambda$ and $\mathrm{q}=1-\frac{\lambda}{n}$.

A random variable $\mathrm{X}$ has a Poisson distribution and it is referred to as Poisson random variable, if and only if its $\mathrm{f}(\mathrm{x})=\mathrm{P}(\mathrm{x} ; \lambda)=\frac{e^{-\lambda} \lambda^{x}}{x !}$ for $\mathrm{x}=0,1,2$. Where, e: a constant equal to approximately 2.71828 ( $\mathrm{e}$ is the base of natural logarithm). $\lambda$ : the mean number of successes that occur in a specified region, $\mathrm{n}$ : the total number of trials, $\mathrm{P}$ : the probability of success for each trial, r: no. of occurrence of a given event (Upadhyay \& Dhakal, 2013).

\section{Real World Application of Poisson Distribution}

- The number of mutations on a given strand of DNA per time unit.

- The number of bankruptcies that are field in a month. 
- The number of network failures per day.

- The number of file server virus infection at a data center during a 24 hour period.

- The number of Airbus 330 aircraft engine shutdowns per 1000000 flight hours.

- The number of hungry persons entering McDonald's restaurant.

- The number of asthma patient arrivals in a given hour at a walk in clinic.

- The number of work related accidents over a given production time.

- The number of birth, deaths, marriages, divorces, suicides and homicides over a given period of time.

\section{Properties of Poisson Distribution}

- Since the variable $X$ can take only integral values $0,1,2, \ldots \ldots \infty$, it is a discrete probability distribution.

- It is known as a uniparametric distribution as it is characterized by only one parameter. If we know $\lambda$ all the probabilities of the Poisson distribution can be obtained.

- Mean of Poisson distribution is $\lambda$.

- The variance of Poisson distribution is $\sigma^{2}=\lambda$. Hence, mean and variance is same in the distribution i.e. mean $=\lambda=$ variance.

- Like binomial distribution, Poisson distribution could also be unmodal or bimodal depending on the values of the parameter $\lambda$.

- $\quad$ Skewness $=\frac{1}{\sqrt{\lambda}}$ and Kurtosis $=\frac{1}{\sqrt{\lambda}}$.

- Poisson distribution is applied when the total number of events is very large but the probability of occurrence is very small i.e. $\mathrm{np}<5$ or $\mathrm{n} \mathrm{q}<5$. So Poisson distribution is used in waiting time problems, Insurance, Physics, biology, in the analysis of rare disease, business economic and industry.

Example of use of Poisson distribution is suppose the number of accidents in the street of Kathmandu follows Poisson distribution with a mean of 3 accidents per day then finding the probability that i. Number of accident in a day ii. Number of accident in a day exceeds 3. Mean of Poisson distribution, $\lambda=3$ accidents per day. Let the random variable $\mathrm{X}$ denotes in the street of Kathmandu which follows 
Poisson distribution with parameter $(\lambda)$ i.e. $\mathrm{X} \sim \mathrm{P}(\lambda)$ where $\lambda=$ mean $=3$ accidents per day. From Poisson distribution the probability that exactly $\mathrm{x}$ accidents occurs in a day is given by $\mathbf{P}(\mathbf{X}=\mathbf{x})=\frac{e^{-\lambda} \lambda^{x}}{x !}=\frac{e^{-3} 3^{x}}{x !}, \mathbf{x}=1,2 \ldots . \infty$.

i. The probability that no accidents occurs in a day is

$$
\mathrm{P}(\mathrm{X}=0)=\frac{e^{-3} 3^{0}}{o !}=e^{-3}=0.04979 .
$$

ii. The probability that number of accidents in a day exceeds 3 is

$$
\begin{aligned}
& \mathrm{P}(\mathrm{X}>3)=1-\mathrm{P}(\mathrm{X}=3)=1-\mathrm{P}(\mathrm{x}=0 \text { or } \mathrm{x}=1 \text { or } \mathrm{x}=2 \text { or } \mathrm{x}=3) \\
& =1-\mathrm{P}(\mathrm{x}=0)+\mathrm{P}(\mathrm{x}=1)+\mathrm{P}(\mathrm{x}=2)+\mathrm{P}(\mathrm{x}=3)=1-e^{-3}\left[1+\frac{3}{1 !}+\frac{3^{2}}{2 !}+\frac{3^{3}}{3 !}\right] \\
& =1-0.049797[1+3+4.5+4.5]=1-0.4979 \mathrm{x} 13=0.35273 .
\end{aligned}
$$

Example of fitting a Poisson distribution using following data:

\begin{tabular}{|l|l|l|l|l|l|}
\hline No. of mistakes per page & 0 & 1 & 2 & 3 & 4 \\
\hline No. of times mistake occurred & 211 & 90 & 19 & 5 & 0 \\
\hline
\end{tabular}

Calculation of mean

\begin{tabular}{|l|l|l|}
\hline No. of mistakes pages(X) & Frequency(f) & $\mathrm{fx}$ \\
\hline 0 & 211 & 0 \\
\hline 1 & 90 & 90 \\
\hline 2 & 19 & 38 \\
\hline 3 & 5 & 15 \\
\hline 4 & 0 & 0 \\
\hline & $\mathrm{N}=325$ & 143 \\
\hline
\end{tabular}

Mean of the Poisson distribution $=143 / 325=0.44$

We know that Parameter of Poisson distribution $=$ mean of the Poisson distribution $=\lambda=0.44$. We have Poisson distribution, $\mathrm{P}(\mathrm{X})=\frac{e^{-\lambda} \lambda^{x}}{x !}=\frac{e^{-0.44}(0.44)^{x}}{x !}$, 
$x=0,1,2,3,4$.Theoretical frequency of the Poisson distribution is given by $f(x)=N . P(x)$, where $\mathrm{N}=$ total frequency.

Substitute $\mathrm{X}=0,1,2 . ., 4$ we get, $\mathrm{P}(\mathrm{o})=\frac{e^{-0.44}(0.44)^{0}}{0 !}=0.6440, \mathrm{P}(1)=\frac{e^{-0.44}(0.44)^{1}}{1 !}$ $=0.2833, \mathrm{P}(2)=\frac{e^{-0.44}(0.44)^{2}}{2 !}=0.0623, \mathrm{P}(3)=\frac{e^{-0.44}(0.44)^{3}}{3 !}=0.0091, \mathrm{P}(4)=$ $\frac{e^{-0.44}(0.44)^{4}}{4 !}=0.0409$.

Calculation of theoretical frequency of the Poisson distribution

\begin{tabular}{|l|l|l|l|}
\hline Mistakes per page & No.(f) & $\mathrm{P}(\mathrm{x})$ & $\begin{array}{l}\text { Theoretical frequency } \\
\text { i.e. N.P(x)=325.P(x) }\end{array}$ \\
\hline 0 & 211 & 0.6440 & 209 \\
\hline 1 & 90 & 0.2833 & 92 \\
\hline 2 & 19 & 0.0623 & 20 \\
\hline 3 & 5 & 0.0091 & 3 \\
\hline 4 & 0 & 0.0409 & 13 \\
\hline & $\mathrm{N}=325$ & & \\
\hline
\end{tabular}

The fitted Poisson distribution is

\begin{tabular}{|l|l|l|l|l|l|}
\hline $\mathrm{r}$ & 0 & 1 & 2 & 3 & 4 \\
\hline $\mathrm{E}$ & 209 & 92 & 20 & 3 & 13 \\
\hline $\mathrm{O}$ & 211 & 90 & 19 & 5 & 0 \\
\hline
\end{tabular}

\section{Normal Distribution}

The normal distribution is also known as the Gaussian distribution which is the most important continuous probability distributions. The normal distribution was first discovered by De Moivre and later rediscovered by Laplace and Gauss. The normal distribution is the limiting form of the binomial distribution when the number of cases of success is infinitely large and probabilities of success and failure are almost equal. In other words, it is a way of assigning probabilities to intervals of real numbers associated 
with continuous random variables. The probabilities are assigned by means of a special curve called the normal curve whose equation often called the probability density function (p.d.f) is $\mathbf{f}(\mathbf{x})=\frac{1}{\sigma \sqrt{2 \pi}} e^{-\frac{1}{2}}\left(\frac{X-\mu}{\sigma}\right),-\infty<\mathbf{X}<\infty$

(I) where $\mu$ and $\sigma$ are parameters, $\Pi=$ constant $=3.14$, e $=$ constant $=$ the base of the natural logarithms $=2.71828$.

\section{Properties of Normal Distribution}

- The curve is bell shaped and symmetrical about the line $X=\mu$ (i.e $z=0$ ) so that $\beta_{1=0}$ and $\Upsilon_{1=0 \text { and }}, \beta_{2=3}, \Upsilon_{2=0}$.

- Mean, median and mode of the distribution coincide.

- Area under the normal curve is unity and the mean divides it into two equal parts.

- The curve has a single peak so it is unimodal.

- $\quad \operatorname{As~} \mathrm{p}(\mathrm{x})$ is the probability, it can never be negative, so no portion of the curve lies below the $\mathrm{X}$-axis.

- The two tails of the normal cure extended indefinitely and never touch the horizontal axis.

- Points of inflexion (the point at which the curve changes its deviation) of the normal curve are at $X=\mu \pm \sigma$ i.e. they are equidistant from mean at a distance of $\sigma$.

- A linear combination of independent normal variate is also normal variate i.e. if $X_{1}, X_{2}, X_{3}, \ldots \ldots . X_{n}$, are independent normal variates with means $\mu_{1}, \mu_{2}, \mu_{3} \ldots \ldots . \mu_{n}$ and standard deviation $\sigma_{1}, \sigma_{2,}, \sigma_{3}, \ldots \ldots \sigma_{n}$, respectively then their linear combination $a_{1} x_{1},+a_{2} x_{2},+a_{3} x_{3}, \ldots \ldots a_{n} x_{n}$, where $a_{1}, a_{2}, \ldots a_{n}$ are consants is also normal variate with mean $=a_{1} \mu_{1}+a_{2} \mu_{2} \ldots .+a_{n} \mu_{n}$, variate $\left(\sigma^{2}\right)=a_{1}^{2} \sigma_{1}^{2}+a_{2}^{2} \sigma_{2}^{2}+\cdots \ldots+a_{n}^{2} \sigma_{n}^{2}$. Thus the sum or difference of independent normal variate is a normal variate.

- Normal distribution is a limiting case of binomial and Poisson distribution.

- In a normal distribution the quartiles ( first and third) are equidistant from median i.e. $Q_{3}+Q_{1}=2 \mathrm{Md}=2 \mu$ 
- In a normal distribution, quartile deviation is approximately equal to $2 / 3$ times its standard deviation and mean deviation about mean is approximately equal to $4 / 5$ times its standard deviation.

- Area property: The most fundamental property of normal probability curve is its area property. The area under normal probability curves between the ordinates $X=\mu-\sigma$ and $X=\mu+\sigma$ is 0.6826 . In other words the range $\mu \pm \sigma$ covers $68.26 \%$ of the observations or $\mathrm{P}(\mu-\sigma<\mathrm{X}<\mu+\sigma)=0.6826, \mathrm{P}(\mu-2 \sigma<\mathrm{X} \mu+2 \sigma)=$ $0.9544, \mathrm{P}(\mu-3 \sigma<\mathrm{X} \mu+3 \sigma)=0.9974$ which is very close to unity.

Because of the discrete random phenomenon, binomial and poisson are discrete probability distribution where as normal distribution is continuous theoretical distribution in statistics. Most of the statistical data in our society relating to economic and business, rural development even in social and physical science confirm to continuous probability distribution which is the most important probability models in the statistical analysis of data (Upadhya \& Dhakal, 2013).

\section{Normal Curve and its Explanation}

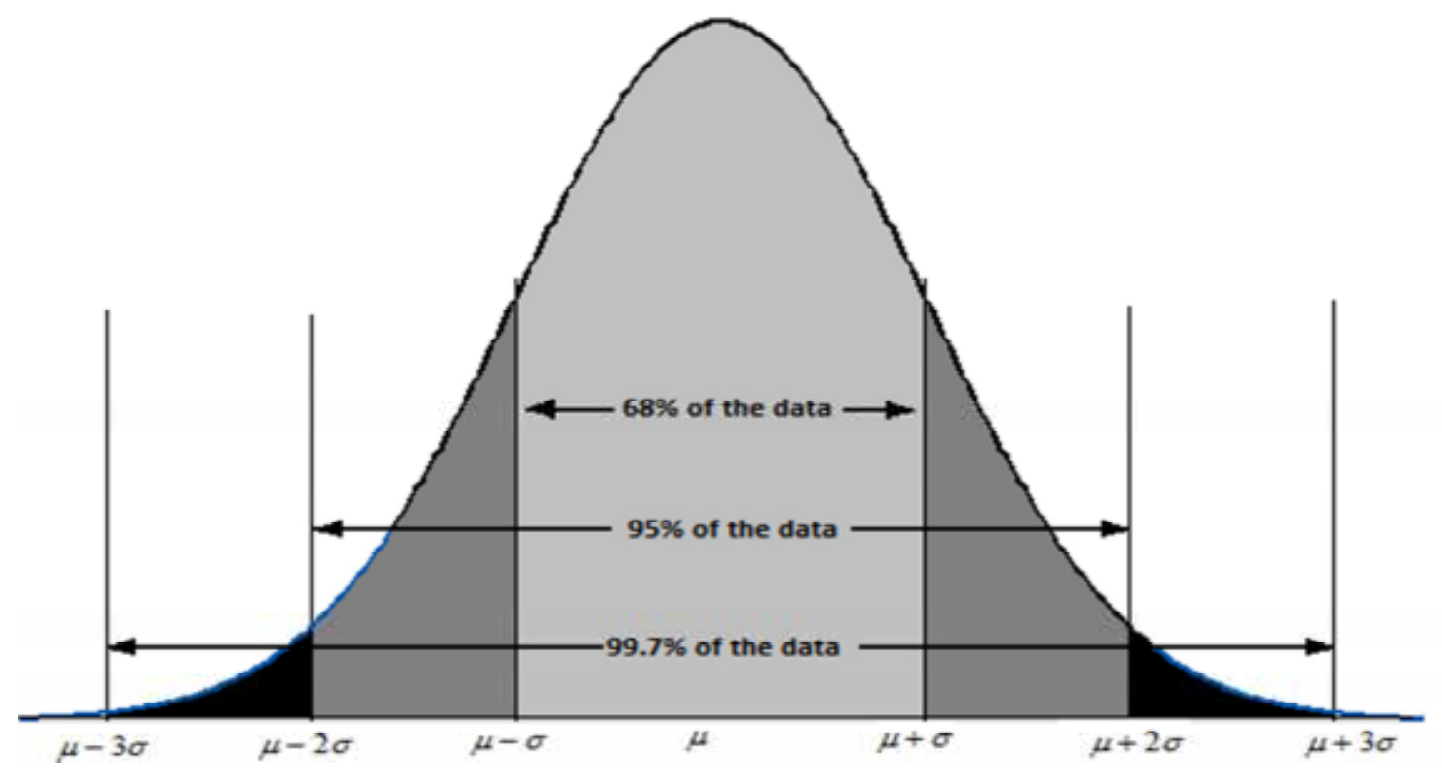

Fig A 
There is a graph of $f(x)$ in Figure A for values of $x$ between $\mu-3 \sigma$ and $\mu+3 \sigma$. It is a symmetric bell shaped curve which slopes downward on both sides from the maximum value (which always occur at the point where $X=\mu$ ) towards the $X$-axis but never touches the X-axis. In this case

Mean $=$ median $=$ mode, mean $=\mu$, standard deviation $=\sigma$, skewness $=0$, kurtosis $=0$ $\left(\beta_{2}=3\right)$ i.e. the normal curve is miso kurtic and it is unimodal i.e. the normal curve has only one mode.

If two populations defined by equation I with different means that is $\mu_{1} \neq \mu_{2}$ but identical standard deviations i.e. $\sigma_{1}=\sigma_{2}$, their graphs would appear like in fig b. If the populations had identical means i.e. $\mu_{1}=\mu_{2}$ but different standard deviation i.e. $\sigma_{1} \neq$ $\sigma_{2}$, the graph would appear as in fig a. If both the means and standard deviations are different we would have curves like in fig c. So the curve becomes flatter and broader with the larger values of $\sigma$. In all these diagrams, the area under the curves and the Xaxis is 1 i.e. $\int_{-\infty}^{\infty} f(x) d(x)=1$.

In fig $b$ and $c$ there is difference in the spread of the curves due to the difference in $\sigma$ 's. Thus a normal distribution is completely characterized by parameters $\mu$ and $\sigma$. The figure is given below:

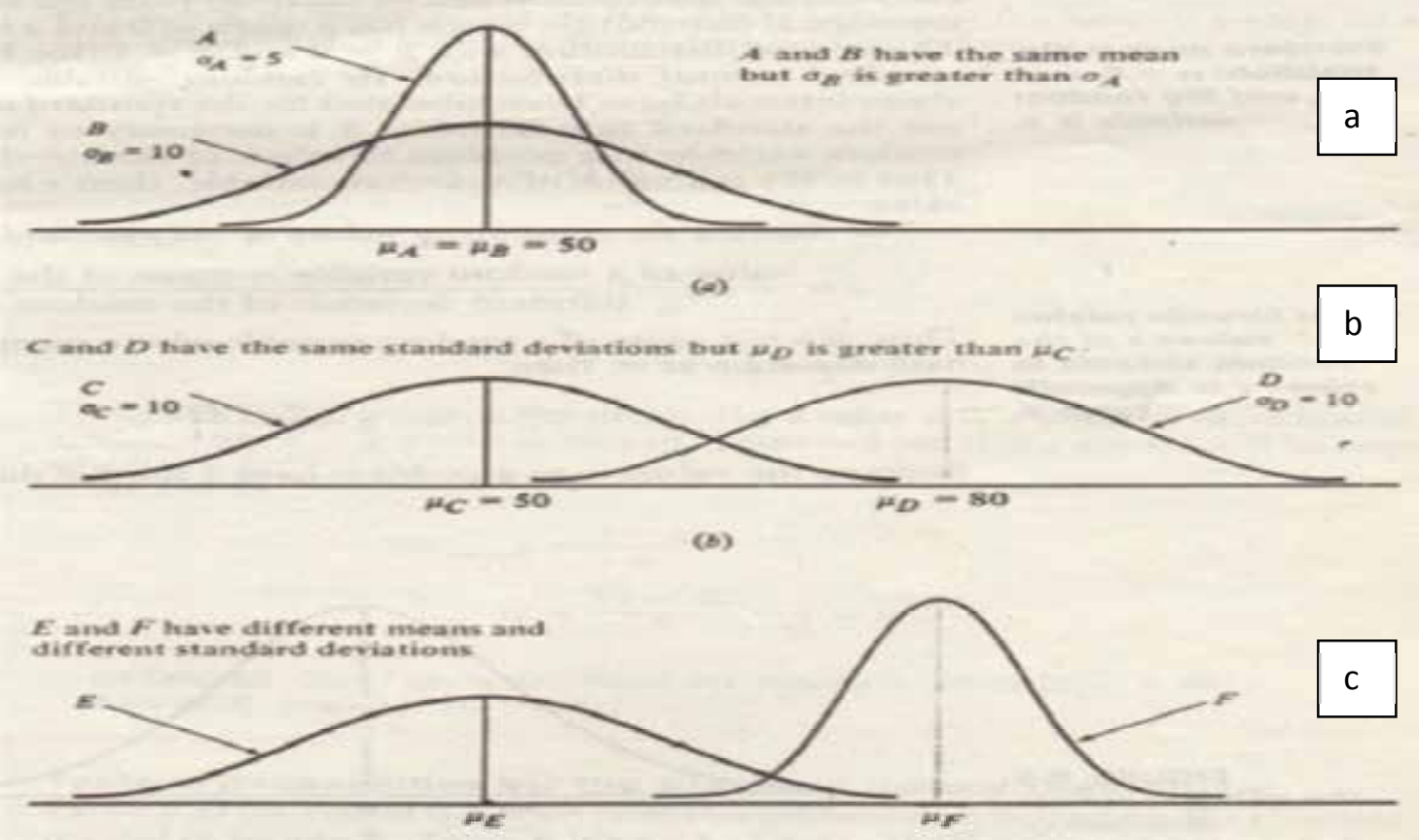




\section{Characteristics of the Normal Probability distribution}

- Each specific normal distribution is differentiated by its mean $\mu$ and its standard deviation $\sigma$.

- The highest point on the normal curve occurs at the mean which is also the median and mode of the distribution.

- The mean of the distribution can be numerical values: negative, zero or positive. For e.g. three curves with the same standard deviation but different means 10, 0 and 20 has been shown below:

- The normal probability distribution is symmetric with the shape of the curve to the left of the mean a mirror image of the shape of curve to the shape of curve to the right of the mean. The tails of the curve extend to infinity in both directions and theoretically never touches the horizontal axis.

- The standard deviation determines the width of the curve. Larger the values of the standard deviations result in wider, flatter curves showing more dispersion in the data.

- The total area under the curve for the normal distribution is 1.(This is true for all continuous probability distributions).

\section{Conclusion}

The study attempts to analyze the basic concept and application of probability distribution. Normal distribution describes continuous data which have a symmetric distribution, with a characteristic 'bell' shape. Binomial distribution describes the distribution of binary data from a finite sample. Thus it gives the probability of getting $" r$ " events out of " $n$ " trials. Poisson distribution describes the distribution of binary data from an infinite sample. Thus it gives the probability of getting " $r$ " events in a population. Hence, from the above study we can gain certain information of probability distribution and its application. 


\section{References}

Ash, R. B. (2008). Basic probability theory. Mineola: Dover Publications, New York, United States.

Feller, W. (1968). An introduction to probability theory and its applications (Third edition). London: Wiley Publication, United Kingdom.

Kolmogorov, A. N. (1956). Foundations of the theory of probability (second edition). University of Oregon: Chelsea Publishing Company, New York, United States.

Khuri, A. I. (2004). Applications of Dirac's delta function in statistics. International Journal of Mathematical Education in Science and Technology, 35(2), 185-195. https://doi: 10. 1080/00207390310001638313.

Michael, E. (2010). Probability and statistics: The science of uncertainty (Second edition).Rosenthal: Jeffrey S. (Jeffrey Seth), W.H. Freeman and Co., New York. United States.

Upadhyay, H. P., \& Dhakal, B. P. (2013). Mathematical statistics. Kirtipur: Sunlight Publication, Kathmandu, Nepal. 